RESEÑA HISTÓRICA

\title{
LABORATORIO DE BROMATOLOGÍA DE FORRAJES CENTRO DE INVESTIGACIÓN EN NUTRICIÓN ANIMAL
}

Adrián J. Martínez-Machado

\section{INTRODUCCIÓN}

En los inicios de 1963 se creó el Laboratorio de Nutrición Animal (LANA), cuando se cerró la cafetería que estaba ubicada en la parte posterior de la Facultad de Agronomía y el Decano, Ing. Salas cedió el espacio para iniciar la instalación del LANA, que sería el primero de esta índole en funcionar en la Facultad. Posteriormente, después de muchas dificultades, en octubre de 1985 se inaugura el Laboratorio de Nutrición Animal en los terrenos de la actual Ciudad de la Investigación de la Universidad de Costa Rica. Posteriormente, el 10 de marzo de 1989, el Consejo Universitario, en su sesión No. 3545, eleva de rango al Laboratorio de Nutrición Animal a Centro de Investigaciones en Nutrición Animal (CINA) (Fonseca, 1992).

El Laboratorio de Forrajes (posteriormente de Bromatología de Forrajes) aparece cuando el LANA se traslada a los terrenos actuales del CINA en 1985. En aquel momento toda la infraestructura y equipos que se utilizaban en el LANA, quedan al servicio de los investigadores de la Escuela de Zootecnia. Este laboratorio tenía la capacidad de secar y moler las muestras de forraje, así como la de realizar tanto el análisis proximal como el fraccionamiento de la fibra de los forrajes, y el análisis de la digestibilidad in vitro de la materia seca.

En 1996, el Laboratorio de Forrajes se traslada al CINA, a un espacio cuatro veces menor al inicial, por lo cual el análisis proximal de los forrajes, es absorbido por el Laboratorio de Química del CINA, sin embargo, por un tiempo se continua analizando Proteína Cruda, y el Laboratorio de Forrajes se renombra como Laboratorio de Bromatología de Forrajes.

Ya instalado en el CINA, el laboratorio de Bromatología de Forrajes continúa con su misión de apoyar a la investigación en forrajes de la Escuela de Zootecnia, el apoyo a la docencia al capacitar sus estudiantes en técnicas de análisis de sus tesis de grado y

\footnotetext{
${ }^{1}$ Universidad de Costa Rica, Facultad de Ciencias Agroalimentarias, Centro de Investigaciones en Nutrición Animal. San José, Costa Rica. Autor para correspondencia: adrian.martinez@ucr.ac.cr

Recibido: 30 de octubre $2015 \quad$ Aceptado: 2 de noviembre 2015
} 
posgrado y el servicio al sector pecuario, brindando los análisis que requieren para sus labores productivas. En cuanto a los equipos, estos paulatinamente se fueron actualizando, para brindar un mejor tiempo de respuesta en los análisis de fraccionamiento de fibra y digestibilidad in vitro de la materia seca. Por otro lado, se incorporaron nuevas metodologías, nacidas de las necesidades de la investigación en nutrición animal.

\section{MATERIALES Y MÉTODOS}

El fraccionamiento de la proteína cruda (PC), permitió desmenuzar el concepto crudo de este análisis de nitrógeno total, y así se pudo conocer qué porcentaje de la proteína cruda correspondía a nitrógeno no proteico de gran solubilidad, que porcentaje correspondía a proteína sobrepasante, que puede ser digerida en el tracto gastrointestinal posterior del animal; cuánto de la PC corresponde a proteína no degradable; que no puede ser metabolizado por el animal, de tal manera que los balances de las dietas y las prácticas de alimentación pueden ser más eficientes, y por ende más económicas.

Los ensilajes son una de las fuentes forrajeras que han representado la respuesta a la necesidad de los productores de utilizar otras fuentes de alimentos y la preservación de los mismos. La fermentación anaeróbica de los forrajes es una de las posibles estrategias para mitigar el efecto estacional de escasez de alimento y el efecto negativo del fenómeno ENOS. El análisis de la calidad de los alimentos ensilados, es importante, pero el análisis de la calidad del proceso de fermentación, lo es también. La posibilidad de conocer el estado fermentativo de los materiales ensilados, sus propiedades fermentativas a priori, permiten al productor mejorar el proceso de ensilado y en gran medida el producto que brindará a sus animales.

Recientemente, se inicia con el análisis de la digestibilidad in vitro de la fibra detergente neutro, este análisis permite al profesional en nutrición animal realizar una mejor estimación de los Nutrimentos Digestibles Totales y de la Energía Neta, así como del potencial de consumo de materia seca por el rumiante, con la consecuente mejora en el balance de la dietas y una producción de leche más predecible. También permite realizar los cambios necesarios en la preparación de las raciones totales, al poder sustituir un 
Martínez-Machado. Historia del Laboratorio de Bromatología del CINA

forraje de baja digestibilidad de la fibra por otro de mejor calidad, agregar otros ingredientes adicionales con una digestibilidad de la fibra alta o cambiar la relación de forrajes en la mezcla.

Además, se incorpora la metodología para la medición de gases de efecto invernadero, el conocimiento de que nivel de producción de dióxido de carbono y metano a nivel ruminal tiene un alimento, puede coadyuvar con el esfuerzo por una ganadería menos contaminante, al utilizar forrajes menos productores de gases y dietas más eficientes, conjuntamente con otras prácticas mitigantes a nivel de finca.

La investigación que se realizó el siglo pasado en el laboratorio se enfocó principalmente en la determinación de la calidad nutricional de los forrajes que en ese momento eran de mayor utilización en el país. La determinación del contenido de proteína cruda, extracto etéreo, cenizas, minerales, el fraccionamiento de la fibra, el nitrógeno ligado a la fibra, la digestibilidad in vitro de la materia seca permite la estimación de la energía de los pastos. Esto permite la estimación de la producción de leche a partir del contenido de proteína o de energía, demostrándose que los pastos tropicales son deficientes en energía, por lo cual es necesario suplementar con fuentes energéticas, para así aprovechar el contenido proteínico de los mismos y lograr un incremento en la producción lechera.

Además, se hicieron importantes descubrimientos acerca de la enfermedad metabólica de la fiebre de leche, tanto las implicaciones sanitarias del animal como las pérdidas económicas del productor, esto permitió brindar recomendaciones tanto en nutrición como en manejo de los animales para disminuir la incidencia de esta enfermedad. En relación con esta enfermedad, se investigó la importancia del balance catión:anión en la dieta de la vaca lechera, por lo que se pudo enmendar las dietas para minimizar el efecto en la incidencia de la fiebre de leche. Este investigación dio pie para la implementación de prácticas de alimentación, nuevos productos para disminuir el efecto de la nutrición mineral en la incidencia de la fiebre de leche y la conciencia en el productor de la importancia de prevenir la aparición de fiebre subclínica en las vacas lecheras.

Por otro lado, se tuvo incidencia en la conservación de la cuenca del río Picagres, en Puriscal; mediante el uso de pasturas mejoradas y prácticas de manejo de potreros, control de carga animal, esto hizo más eficiente el uso de los potreros, permitiendo la segregación de áreas para reforestación y la disminución de la erosión del suelo. En esta 
misma zona se determinó la calidad de las leguminosas presentes en la zona, como parte del manejo integral de las pasturas.

La calidad nutricional y el efecto de ensilado de la Cratylia argentea, también fue parte de la investigación del laboratorio, conjuntamente con el INTA, en la búsqueda de nuevas fuentes forrajeras en el Pacífico nacional.

En este siglo, la investigación se enfocó en la composición nutricional de otras fuentes no forrajeras, tales como los subproductos húmedos agroindustriales: banano, plátanos, piña y otras frutas; los subproductos de agroindustriales fibrosos, tales como: subproductos del arroz y el maíz: tamo, semolina, puntilla de arroz y salvadillo; el subproducto de la producción de cervecería como los residuos de cebada. Con esto se generó información que permitió el balanceo de dietas para el consumo animal utilizando estos subproductos para disminuir el consumo de maíz y harina de soya, y facilitar la utilización de estos desechos, disminuyendo los costos de producción y la contaminación del ambiente.

La determinación del contenido en sangre de vitamina $E$, calcio y fósforo en las vacas en el periparto, mostró que vacas consumiendo pastos verdes, como sucede en nuestro país, no presentaban una disminución en sangre del nivel de vitamina $E$ por debajo del nivel crítico, como si lo presentan los animales que no pastorean, hecho desconocido en su momento por los investigadores de latitudes en que generalmente las vacas lecheras tienen un acceso limitado a pasturas verdes.

Además se participó en conjunto con la Cámara de Ganaderos de la Zona Sur, en un proyecto que determinó la calidad nutricional de los pastos presentes en las fincas que participaron del proyecto en la Zona Sur; aunado al estudio de la relación entre la nutrición mineral y la reproducción del ganado de carne. Con esta investigación se determina que estos pastos eran deficientes en selenio, calcio, fósforo, y que era necesario la suplementación mineral de los animales para mejorar los índices reproductivos en esta zona.

Posteriormente, la investigación se enfocó en la determinación de la composición botánica, producción de biomasa, calidad nutricional y fenología de los principales pastos utilizados en la producción lechera nacional, tales como Rye grass, Kikuyo, Phalaris sp. y Estrella Africana. El objetivo fue establecer el mejor momento para la cosecha del follaje de las pasturas, según su fenología; estableciendo en que momento del crecimiento de la 
Martínez-Machado. Historia del Laboratorio de Bromatología del CINA

planta, según la cantidad de hojas, tiene la mejor concentración de nutrimentos, para ofrecer al animal, esto implica un mejor manejo de las pasturas, permitiendo mayor eficiencia en la rotación de los potreros.

En el año 2014 se inicia la recopilación de la información nutricional de los forrajes analizados por el Laboratorio de Bromatología de Forrajes, bajo el proyecto de investigación No. 739-B4-130. Se ha tabulado la información desde el año 2003, un total de 2041 forrajes, en el que un 63\% de la información corresponde a pastos y un $22 \%$ a ensilajes, el detalle se puede apreciar en el Cuadro 1.

Cuadro 1. Distribución de los forrajes tabulados desde el año 2003 que han sido analizados en el Laboratorio de Bromatología de forrajes.

\begin{tabular}{lc}
\hline \multicolumn{1}{c}{ Tipo de forraje } & Cantidad \\
\hline Pastos & 1294 \\
Leguminosas & 53 \\
Arbustivas & 85 \\
Ensilajes & 453 \\
Henos & 90 \\
Subproductos agroindustriales húmedos & 19 \\
Otros & 47
\end{tabular}

La mayor proporción de pastos analizados desde el año 2003 corresponden en un 28\% a especies del género Brachiaria, un 14\% al pasto Kikuyo y un $13 \%$ al pasto Estrella Africana, el $45 \%$ restante corresponden a una serie de pastos naturales y algunos pastos de altura como Rye grass y Phalaris sp., en el Cuadro 2 se denota la distribución de los pastos con mayor representación en la tabla de composición nutricional. 
Cuadro 2. Distribución de los principales pastos que han sido analizados en el Laboratorio de Bromatología de Forrajes cuya información ha sido tabulada desde el año 2003.

\begin{tabular}{lc}
\hline \multicolumn{1}{c}{ Pasto } & Cantidad \\
\hline Género Brachiaria & 363 \\
Kikuyo & 188 \\
Estrella Africana & 167 \\
Phalaris sp. & 144 \\
Ryegrass & 123 \\
Pennisetum purpureum & 112 \\
Guinea & 34 \\
\hline
\end{tabular}

Además de pastos, existe información de ensilajes, leguminosas, plantas arbustivas, henos, y en menor medida de subproductos agroindustriales húmedos, mezclas de pastos y otros materiales utilizados en la alimentación animal, como semillas y frutas. En cuanto a los ensilajes, un $21 \%$ de los ensilajes registrados corresponden a ensilaje de maíz, un $15 \%$ a ensilajes de maíz con alguna especie de leguminosa, un $12 \%$ a ensilajes compuestos principalmente de residuos de piña, y el 52\% restante incluye ensilajes de pastos, ensilaje de caña de azúcar más otros materiales, ensilaje de sorgo, ensilaje de guineo, ensilaje de yuca y en menor porcentaje de ensilajesde algunas leguminosas.

La información almacenada de heno corresponde en un $60 \%$ a heno de trasvala y el $40 \%$ restante se distribuye principalmente en henos de otros pastos y algunas leguminosas como Arachis pintoi. En el caso de la información registrada de leguminosas en la tabla, un 55\% corresponde a Cratylia argentea, un $19 \%$ a Stylosanthes guianensis y en menor cantidad Arachis pintoi, poró, trébol blanco, kudzu. Finalmente, en la información recopilada de plantas arbustivas y árboles que se utilizan para consumo animal, un 33\% corresponde a Morera, un $32 \%$ de follaje de Guácimo y un $25 \%$ de follaje de Jocote. Este es un trabajo que se mantiene en constante captación de información y actualización.

En 2014, también se inicia con la creación de ecuaciones de predicción de composición nutricional en forrajes, a través de la espectroscopia del infrarrojo cercano, bajo el proyecto de investigación 739-B4-223. Se escanean 307 muestras de forrajes, principalmente pastos. Hasta el momento se desarrollan 232 ecuaciones, se prueban 6 
diferentes correcciones matemáticas, estas se calibran y validan para Fibra detergente neutro tratada con amilasa, de las cuales 12 de las ecuaciones tienen un valor predictivo aceptable (RPD cercano a 3), con un error estándar de calibración de 2.5, un error estándar de validación cruzada de 3.1, un error estándar de predicción de 2.9 y un coeficiente de correlación de 0.9; valores propios de la predicción por NIRS de forrajes, debido a la complejidad de las muestras y este caso a la poca variabilidad.

\section{CONSIDERACIONES FINALES Y APLICACIONES}

La presencia de taninos en leguminosas es un hecho conocido, su efecto en la nutrición animal es de tomarse en cuenta, principalmente cuando se utilizan estos forrajes en las prácticas de alimentación. Los taninos afectan el consumo de alimento, la digestibilidad de la ración, y por ende, la eficiencia de la producción. Los efectos varían según el contenido de taninos en el alimento, el tipo de taninos y la tolerancia del animal. Los taninos tienen la capacidad de ligarse a las proteínas y a las fibras, por lo cual reducen la digestión de ambas. La implementación de metodologías para el análisis de los diferentes tipos de taninos y su efecto en la digestibilidad es necesario, para realizar los ajustes necesarios de las dietas que se balancean para animales rumiantes.

Es también requerido continuar con el desarrollo de ecuaciones para los diferentes nutrimentos, e incorporar nuevos espectros para ampliar la variabilidad del colectivo de forrajes utilizados en nuestro país.

\section{LITERATURA CONSULTADA}

Hernán Fonseca Zamora. 1992. Carta del 24 de noviembre de 1992 al señor Director del CINA, Dr. José Ramón Molina. 